OPEN

SUBJECT AREAS:

ANTIVIRALS

RNA-BINDING PROTEINS

Received

29 August 2013

Accepted

7 April 2014

Published

23 April 2014

Correspondence and requests for materials should be addressed to M.W.P. (mparker@svi. edu.au)

\section{Potent hepatitis $C$ inhibitors bind directly to NS5A and reduce its affinity for RNA}

\author{
David B. Ascher', Jerome Wielens' ${ }^{1,2}$, Tracy L. Nero', Larissa Doughty' , Craig J. Morton' \\ \& Michael W. Parker ${ }^{1,3}$
}

\begin{abstract}
${ }^{1}$ ACRF Rational Drug Discovery Centre and Biota Structural Biology Laboratory, St Vincent's Institute of Medical Research, 9 Princes Street, Fitzroy, Victoria 3056, Australia, ${ }^{2}$ Department of Medicine, University of Melbourne, 41 Victoria Parade, Fitzroy, Victoria 3065, Australia, ${ }^{3}$ Department of Biochemistry and Molecular Biology, Bio2 1 Molecular Science and Biotechnology Institute, University of Melbourne, Parkville, Victoria 3052, Australia.
\end{abstract}

Hepatitis $\mathrm{C}$ virus (HCV) infection affects more than 170 million people. The high genetic variability of HCV and the rapid development of drug-resistant strains are driving the urgent search for new direct-acting antiviral agents. A new class of agents has recently been developed that are believed to target the HCV protein NS5A although precisely where they interact and how they affect function is unknown. Here we describe an in vitro assay based on microscale thermophoresis and demonstrate that two clinically relevant inhibitors bind tightly to NS5A domain 1 and inhibit RNA binding. Conversely, RNA binding inhibits compound binding. The compounds bind more weakly to known resistance mutants L31V and Y93H. The compounds do not affect NS5A dimerisation. We propose that current NS5A inhibitors act by favouring a dimeric structure of NS5A that does not bind RNA.
$\mathrm{H}$ epatitis $\mathrm{C}$ virus (HCV) infection, a major cause of acute hepatitis and chronic liver disease, is a widespread problem (http://www.who.int). Although the current standard of care, a combination of pegylated interferon, ribavirin and a protease inhibitor ${ }^{1}$, has improved efficacy over earlier treatments ${ }^{2,3}$ it continues to suffer from limitations including long length of treatment and additional side effects ${ }^{1-3}$. An entirely new class of agents exemplified by BMS-790052 (daclatasvir, Bristol-Myers Squibb, Fig. 1a) ${ }^{4}$ and AZD7295 (AstraZeneca, Fig. $1 \mathrm{~b})^{5}$ are amongst the most potent antivirals ever discovered with broad activity against HCV genotypes and subtypes. Identified from cell-based HCV genotype $1 \mathrm{~b}$ replicon assays they have been described as NS5A inhibitors on the basis of resistance mutants ${ }^{4,6}$, pull-down assays ${ }^{4,7}$ and chemical cross-linking ${ }^{7}$, although there is no confirmed mechanism of action. It is possible that the inhibitors disrupt the interaction of NS5A with other proteins; current estimates put the NS5A interactome at 132 human proteins, demonstrating the importance and variety of protein interactions in NS5A functions ${ }^{8}$.

In HCV infected cells, NS5A is produced as part of the viral polyprotein. Once cleaved from the polyprotein, NS5A localises to membranes where it binds to the $3^{\prime}$-end of newly synthesised viral RNA and participates in genome replication, in part through interactions with the viral RNA-dependent RNA polymerase NS5B $\mathrm{B}^{2,8-10}$. NS5A is a 447 residue RNA binding phosphoprotein consisting of three cytoplasmic domains (Fig. 1c). Across the HCV genotypes, domain 1 is well conserved with two crystal structures of domain 1 revealing the same zincbinding monomer conformation but different dimer organisations (Fig. 1d) ${ }^{10,11}$. Whether either homodimer assembly is physiologically relevant has been the subject of debate $e^{7,11-15}$. Domains 2 and 3, however, are more varied in sequence ${ }^{16}$ and, in isolation, are disordered in solution ${ }^{16-18}$. Domain 1 is preceded by an $\mathrm{N}$-terminal amphipathic $\alpha$-helix that is thought to anchor NS5A to the endoplasmic reticulum-derived membrane ${ }^{10,19}$.

Here we describe the first in vitro NS5A binding assay using a recently developed technique called microscale thermophoresis $(\mathrm{MST})^{20}$, a fluorescence-based method, which can measure the binding of small molecules to proteins in solution. We show that BMS-790052 and AZD7295 bind tightly and specifically to domain 1 of NS5A. Furthermore, we show that both compounds inhibit RNA binding to NS5A, believed to be necessary for HCV replication, and bind significantly more weakly to the common resistance mutants $\mathrm{Y} 93 \mathrm{H}$ and L31V. We demonstrate that the compounds bind to NS5A dimers and describe computational models that explain their mode of inhibition. The in vitro protein assay described here will be a valuable tool in the development of next generation NS5A inhibitors for the treatment of HCV infection. 

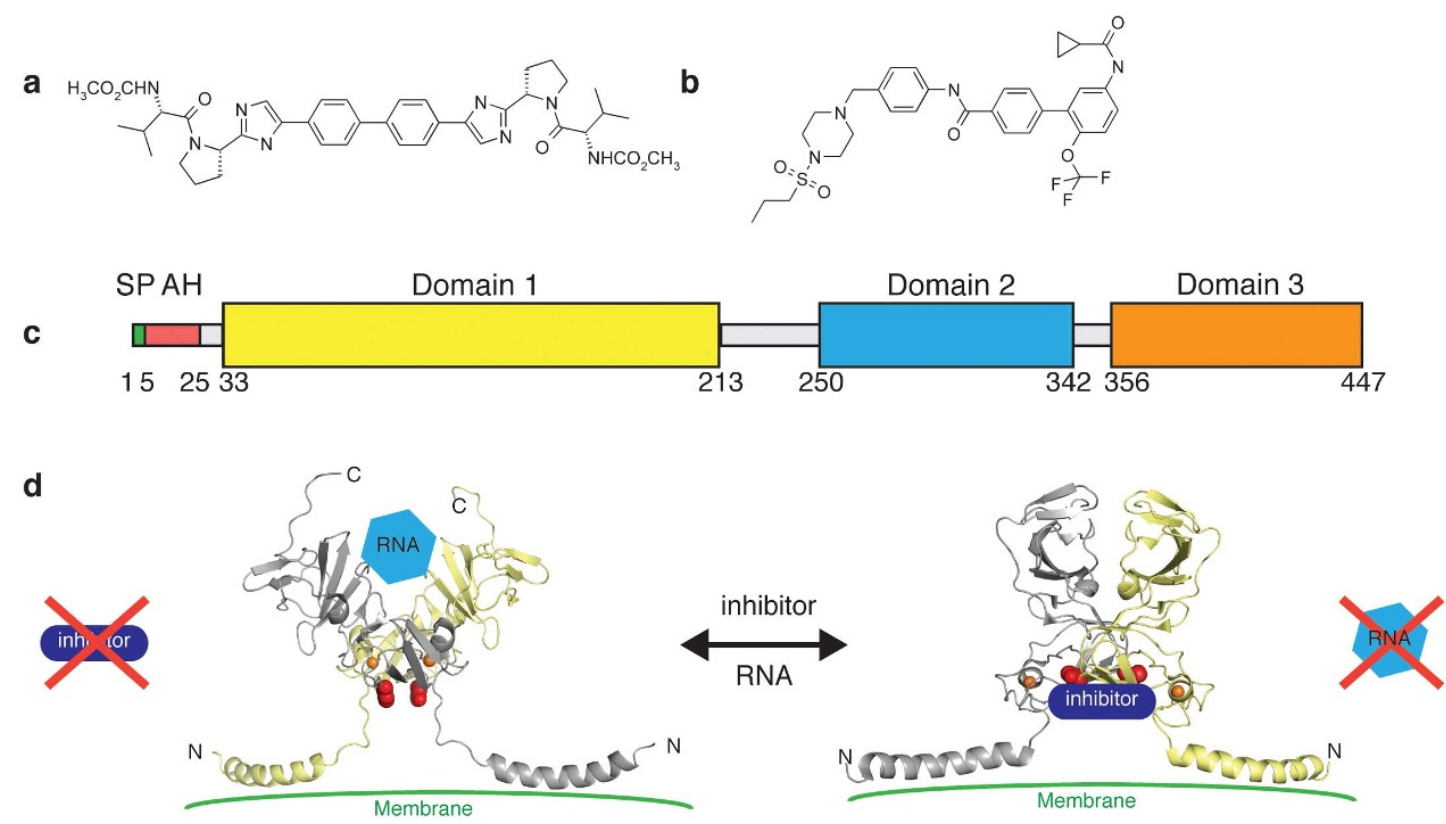

Figure 1 Hepatitis C NS5A and inhibitor structures. Chemical structures of (a) BMS-790052 and (b) AZD7295. (c) NS5A domain structure. SP denotes the signal peptide and AH is an N-terminal amphipathic helix. (d) Proposed mechanism of action of NS5A inhibition. Compound binding to NS5A domain 1 prevents RNA binding. Models of observed NS5 $\mathrm{A}^{1-191}$ dimer conformations (based on crystal structures PDB ID: $1 Z \mathrm{ZH} 1^{10}$ and $3 \mathrm{FQQ}{ }^{11}$ and the NMR structure PDB ID: $1 \mathrm{R} \mathrm{G}^{19}$ ) displayed as cartoon and coloured by monomer. The location of Y 93 in each monomer is indicated by red cpk and the $\mathrm{Zn}^{2+}$ ions are orange spheres.

\section{Results}

In vitro NS5A binding assay. We have used $\mathrm{MST}^{20}$ to develop an in vitro NS5A binding assay. MST data arise from the spontaneous movement of fluorescently labelled protein in a glass capillary tube along induced microscopic temperature gradients generated by an infra-red laser. The thermophoretic movement can be monitored by measuring the local changes in fluorescence intensity emitted from the labelled protein. The rate of this thermophoresis is dependent on a number of factors including the solvent structure around a molecule, and crucially is altered by the binding of a ligand. Measuring the thermophoretic behaviour of a protein in the presence of differing ligand concentrations by MST allows quantitative analysis of molecular interactions in solution on the microlitre scale. The MST technique has previously been used to investigate protein-protein $^{21,22}$, small organic molecule-protein ${ }^{23-25}$ and antibody-protein interactions ${ }^{26}$.

MST was initially used to confirm the interaction of short, single stranded RNA [consisting of four, five or ten uridines $(4 \mathrm{rU}, 5 \mathrm{rU}$ or $10 \mathrm{rU}$ respectively) or five adenines $(5 \mathrm{rA})]$ or double stranded $5 \mathrm{rU}$ $(\mathrm{ds}(5 \mathrm{rU}))$ with NS5A domain 1 . In this study we explored binding to two different NS5A constructs: HCV genotype $1 \mathrm{~b}$ (con1) residues 33-202 (NS5A $\mathrm{A}^{33-202}$ ) and residues 26-202 (NS5A $\left.{ }^{26-202}\right)$. The majority of studies in this work were performed with the former construct, which expresses at relatively high levels and results in a well behaved protein that has been used previously in crystallographic studies ${ }^{11}$. Extended constructs have been reported by others to be very difficult to handle due to the stickiness of the amphipathic N-terminal helix and proteolytic susceptibility of domains 2 and 3 . The NS5A $\mathrm{A}^{26-202}$ construct did not express well and produced a protein that was prone to aggregation and thus difficult to handle. This construct was used to explore one of the documented resistance mutants (L31V) to BMS790052.

The MST data show that NS5A $\mathrm{A}^{33-202}$ binds $4 \mathrm{rU}$ with a $K_{\mathrm{D}}$ of $650 \pm$ $39,5 \mathrm{rU}$ with a $K_{\mathrm{D}}$ of $50 \pm 15,10 \mathrm{rU}$ with a $K_{\mathrm{D}}$ of $20 \pm 9 \mathrm{nM}$ and $5 \mathrm{rA}$ with a $K_{\mathrm{D}}$ of $1.0 \pm 0.2 \mu \mathrm{M}$ (Fig. $2 \mathrm{a}-\mathrm{d}$ ). There was no statistically significant difference between the MST data obtained for RNA binding to the $\mathrm{NS}^{2} \mathrm{~A}^{26-202}$ (Fig. 3a-d, p values of $0.6,0.9,0.9$ and 0.3 for
4rU, 5rU, 10rU and 5rA, respectively) and NS5A $\mathrm{A}^{33-202}$ constructs. The MST data are consistent with published filter-binding and fluorescence polarisation assays for genotype $1 \mathrm{~b}(\operatorname{con} 1)$ NS5A $\left(K_{\mathrm{D}}\right.$ of $600 \pm 200 \mathrm{nM}$ for $4 \mathrm{rU}, 100 \pm 20 \mathrm{nM}$ for $5 \mathrm{rU}, 100 \pm 10 \mathrm{nM}$ for $10 \mathrm{rU}$ and $1000 \pm 200 \mathrm{nM}$ for $5 \mathrm{rA})^{14}$. There was no detectable binding to double stranded $5 \mathrm{rU}$ by any of the NS5A constructs.

Overall, this part of the study showed both NS5A constructs bound various RNA fragments in accord with published values and thus validated the MST assay.

NS5A inhibitors bind to domain 1 of NS5A and compete with RNA binding. We then investigated the binding of BMS-790052 and AZD7295: they both bound tightly to NS5A $\mathrm{A}^{33-202}$ with affinities in the low $n M$ range (Fig. $2 \mathrm{e}, K_{\mathrm{D}}=8 \pm 3 \mathrm{nM}$ and Fig. $2 \mathrm{f}, K_{\mathrm{D}}=30 \pm$

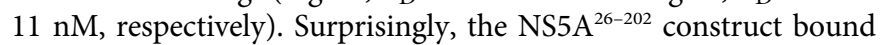
more weakly to BMS-790052 (Fig. $3 \mathrm{e}, K_{\mathrm{D}}$ of $210 \pm 46 \mathrm{nM}$ ) and

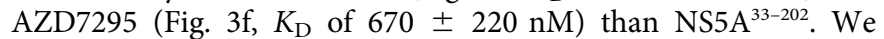
hypothesise that the NS5A N-terminal tail (residues 26 to 32) in the absence of the N-terminal amphipathic helix (residues 5-25) may compete for the same binding site as the inhibitor.

Incubation of $\mathrm{NS} \mathrm{A}^{33-202}$ with 100 -fold excess $5 \mathrm{rU}$ prior to adding compound eliminated binding of both BMS-790052 and AZD7295 (Fig. $2 \mathrm{~g}$ and $2 \mathrm{~h}$ respectively). When NS5A $\mathrm{A}^{33-202}$ was pre-incubated with either $0.25 \mu \mathrm{M}$ BMS-790052 or $0.5 \mu \mathrm{M}$ AZD7295, the affinity of NS5A $\mathrm{A}^{33-202}$ for $5 \mathrm{rU}$ was dramatically shifted from the low $\mathrm{nM}\left(K_{\mathrm{D}}\right.$ $=50 \pm 15 \mathrm{nM}$, Fig. $2 \mathrm{~b})$ to $\mu \mathrm{M}\left(K_{\mathrm{D}}=94 \pm 11 \mu \mathrm{M}\right.$, Fig. $2 \mathrm{i}$ and $K_{\mathrm{D}}=4$ $\pm 1 \mu \mathrm{M}$, Fig. $2 \mathrm{j}$, respectively).

Overall, this part of the study showed unambiguously that both BMS-790052 and AZD7295 bind directly to a site located in NS5A domain 1 with nanomolar affinity. Previously, it was suspected that BMS-790052 bound to domain 1 based on resistance mutations although it couldn't be ruled out that the compound was binding to an interacting protein ${ }^{2,4,6}$. The MST data demonstrate a direct, tight interaction between the compounds and NS5A domain 1 and, most importantly, show that RNA binding inhibits compound binding and, conversely, compound binding inhibits RNA binding. Thus a possible mechanism of action for BMS- 

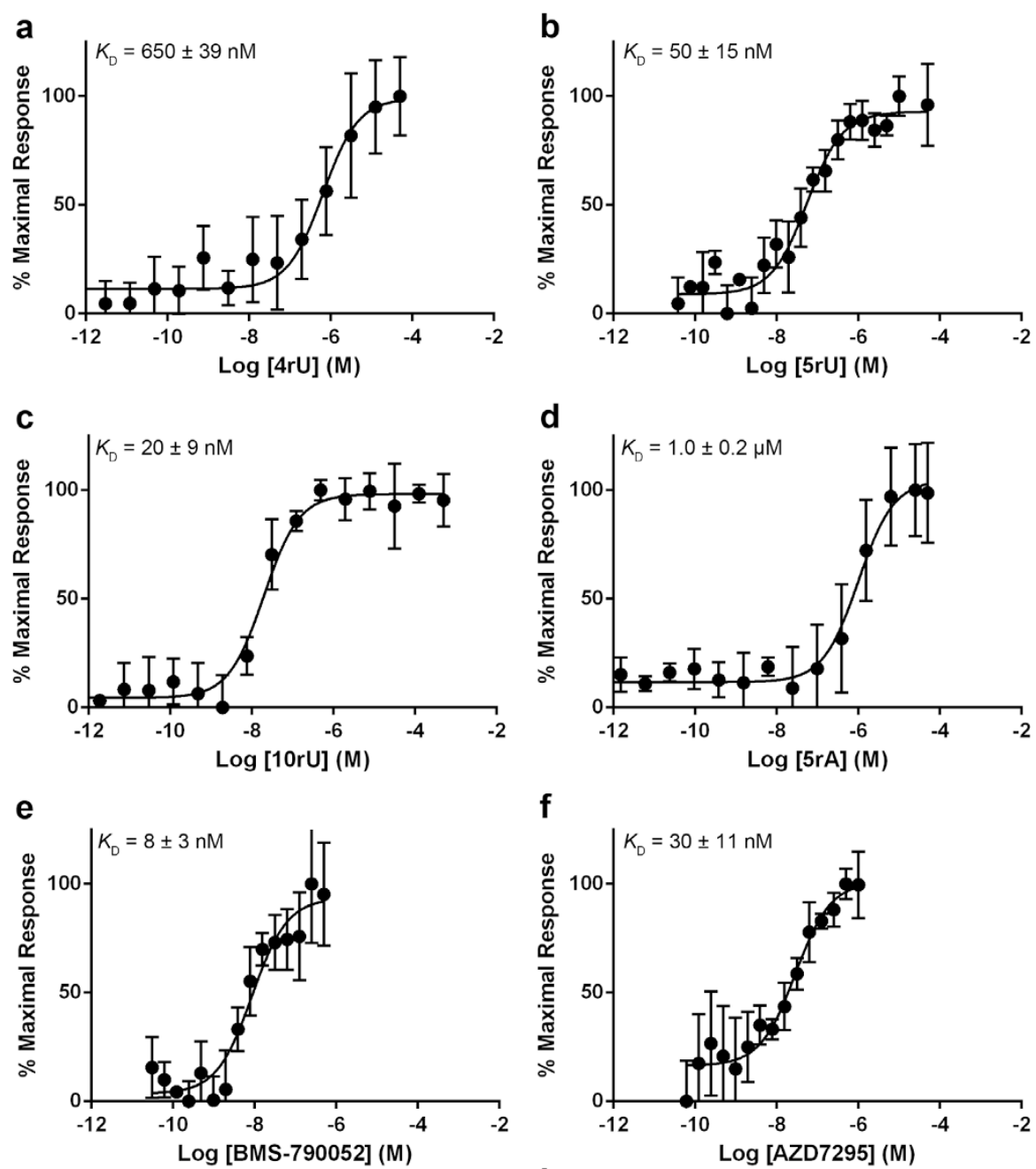

g
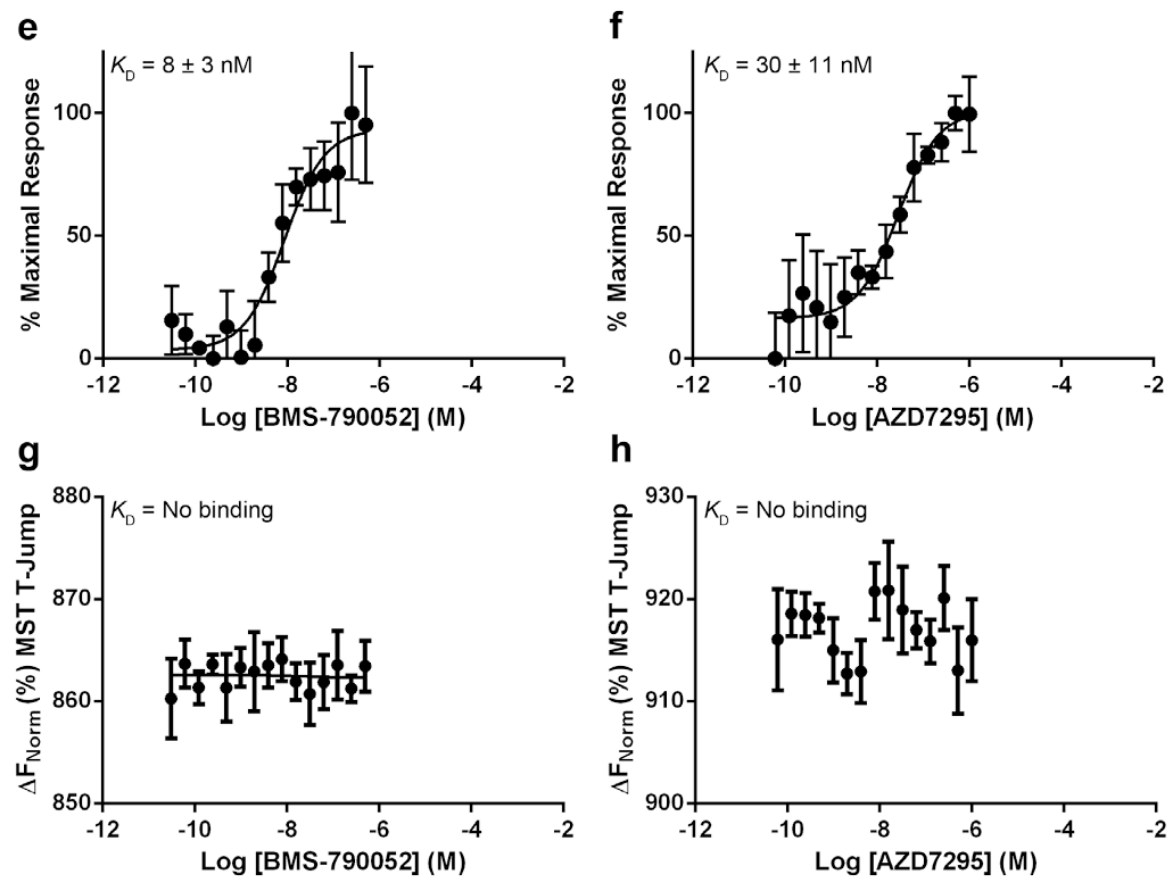

h

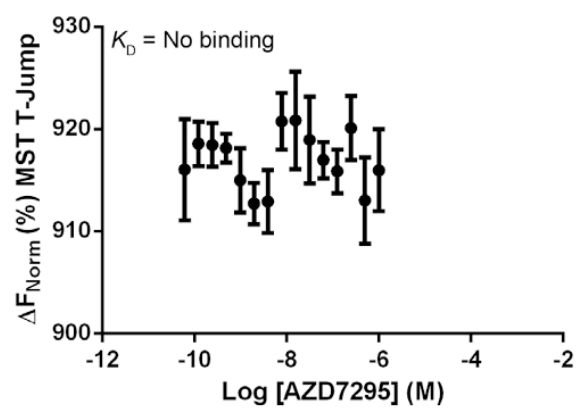

i

j
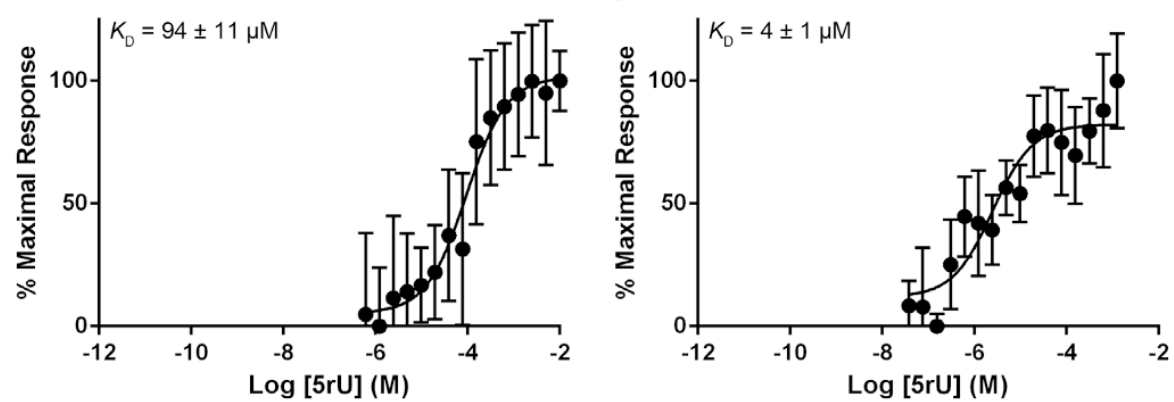

Figure $2 \mid$ Binding to domain 1 NS5A ${ }^{33-202}$ genotype $1 b$ (con1). Binding of (a) 4rU, (b) 5rU, (c) 10rU, (d) 5rA, (e) BMS-790052, (f) AZD7295, (g) BMS-790052 in the presence of 5-fold excess 5rU, (h) AZD7295 in the presence of 5-fold excess 5rU, (i) $5 \mathrm{rU}$ in the presence of $0.25 \mu \mathrm{M}$ BMS-790052, or (j) $5 \mathrm{rU}$ in the presence of $0.5 \mu \mathrm{M}$ AZD7295 to NS5A ${ }^{33-202}$. Values on the Y-axis represent the percentage of the maximal thermophoretic response observed, or in the case of no detectable binding values on the $\mathrm{Y}$-axis represent the thermophoretic shift of the labelled protein. All binding curves were determined in triplicate by MST and are represented as the mean \pm SD. 

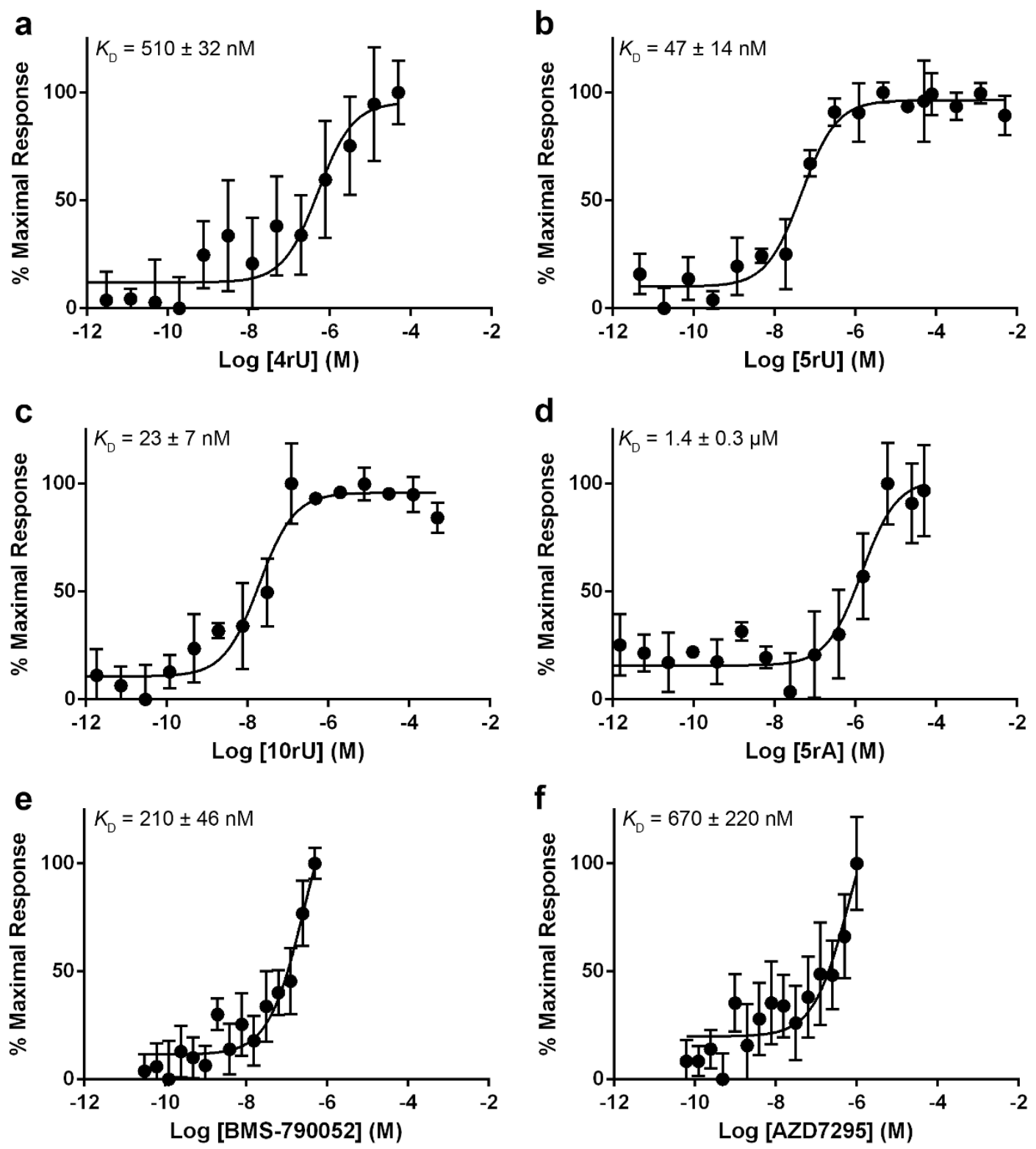

Figure 3 Binding to domain 1 NS5A ${ }^{26-202}$ genotype $1 b$ (con1). Binding of (a) 4rU, (b) 5rU, (c) 10rU, (d) 5rA, (e) BMS-790052 or (f) AZD7295 to $\mathrm{NS}^{2} \mathrm{~A}^{26-202}$. Values on the $\mathrm{Y}$-axis represent the percentage of the maximal thermophoretic response observed. All binding curves were determined in triplicate by MST and are represented as the mean \pm SD.

790052 and AZD7295 is that they directly reduce the affinity of NS5A for viral RNA.

NS5A inhibitors bind more weakly to common resistance mutants. Mechanism of action studies have revealed that a number of mutants conferring resistance against both BMS-790052 and AZD7295 mapped to the NS5A region of the viral genome ${ }^{6,27-29}$. Two of the most common mutation sites are $\mathrm{Y} 93 \mathrm{H} / \mathrm{N} / \mathrm{C}$ and $\mathrm{L} 31 \mathrm{~V} / \mathrm{M} / \mathrm{F}$ which confer 19- to 47,000-fold and 23- to 3,350-fold resistance against BMS-790052, respectively ${ }^{4,6}$. We thus predicted that compound binding to these common resistance mutants would be compromised, but that the mutants would be unlikely to affect RNA binding significantly since that would compromise NS5A function.

Both BMS-790052 $\left(K_{\mathrm{D}}>0.5 \mu \mathrm{M}\right.$; Fig. 4e) and AZD7295 $\left(K_{\mathrm{D}}>\right.$ $1 \mu \mathrm{M}$; Fig. 4f) showed significantly weaker binding to the resistance mutant $\mathrm{Y} 93 \mathrm{H}$ NS5 $\mathrm{A}^{33-202}$, although we were unable to determine a precise $K_{\mathrm{D}}$ due to their limited solubility $(<1.6 \mu \mathrm{g} / \mathrm{ml}$ at $\mathrm{pH} 7.0)$. The L31V NS5A ${ }^{26-202}$ resistance mutation did not show any detectable binding to either inhibitor (Fig. 5e and $\mathrm{f}$ ).
In contrast, RNA binding was unaffected for the L31V NS5A $\mathrm{A}^{26-202}$ construct compared to wild type NS5 $\mathrm{A}^{26-202}$ ( $\mathrm{p}$ values of $0.9,0.3,0.7$ and 0.5 for $4 \mathrm{rU}, 5 \mathrm{rU}, 10 \mathrm{rU}$ and $5 \mathrm{rA}$, respectively, Fig. 5a-d). The Y93H NS5 $\mathrm{A}^{33-202}$ resistance mutation had little effect upon the binding of $4 \mathrm{rU}$ or $5 \mathrm{rU}$ compared to wild type $\left(K_{\mathrm{D}}\right.$ of $1000 \pm 270 \mathrm{nM}$ and $200 \pm 20 \mathrm{nM}$ respectively, Fig. $4 \mathrm{a}$ and b); however, there was a statistically significant difference in the binding of 10rU compared to wild type NS5A $\mathrm{A}^{33-202}$ (Fig. $4 \mathrm{c}, K_{\mathrm{D}}$ of $150 \pm 15 \mathrm{nM}, \mathrm{p}<0.01$ ) and 5rA (Fig. $4 \mathrm{~d}, K_{\mathrm{D}}$ of $2.6 \pm 0.28 \mu \mathrm{M}, \mathrm{p}<0.01$ ).

Overall, this part of the study showed that common resistance mutants to clinically relevant NS5A inhibitors act by directly reducing affinity towards the compounds with little affect on RNA binding.

NS5A inhibitors do not affect NS5A dimerisation. The observation that many NS5A inhibitors are roughly symmetrical has led to suggestions they might bind across the NS5A dimer interface ${ }^{4,7,11,13,15}$. As the presence of NS5A monomers in solution has been reported in the 

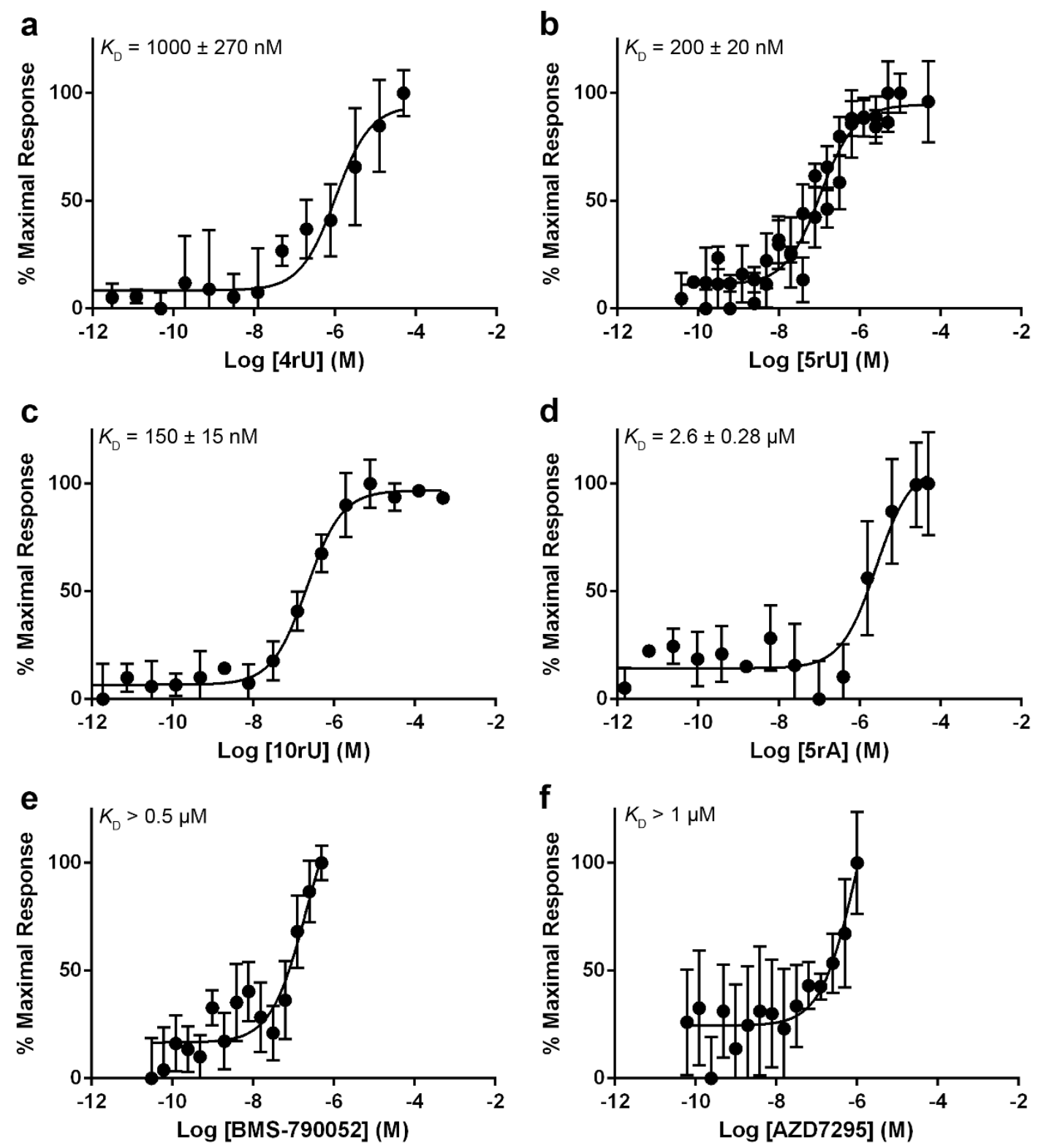

Figure $4 \mid$ Binding to domain 1 NS5A ${ }^{33-202}$ Y93H genotype $1 b$ (con1). Binding of (a) 4rU, (b) 5rU, (c) 10rU, (d) 5rA, (e) BMS-790052 or (f) AZD7295 to $\mathrm{NS}_{5} \mathrm{~A}^{33-202} \mathrm{Y} 93 \mathrm{H}$. Values on the $\mathrm{Y}$-axis represent the percentage of the maximal thermophoretic response observed. All binding curves were determined in triplicate by MST and are represented as the mean \pm SD.

literature ${ }^{14}$, we wanted to explore whether the inhibitors directly bind to NS5A dimers, monomers or both species.

Multi-Angle Laser Light Scattering (MALLS) analysis of the NS5 $\mathrm{A}^{33-202}$ construct shows that it assembles almost exclusively as dimers in solution (Fig. 6a, average molecular weight $39 \pm 5 \mathrm{kDa}$ ). The addition of 5rU, BMS-790052 or AZD7295 did not affect the dimerisation state (Fig. 6b-d, average molecular weights of $38 \pm 6,41$ \pm 7 and $40 \pm 5 \mathrm{kDa}$ respectively). These results agree with previous findings that RNA or compounds do not affect dimerisation ${ }^{14,15}$.

In silico docking of inhibitors to NS5A domain 1 models. Having shown that the inhibitors bind to NS5A domain 1 and that common resistance mutants affect their binding, we wished to define the details of the binding site. Extensive crystallographic studies by us, and others, have failed to observe the direct binding of inhibitors to the protein. Hence as a first step we computationally docked the inhibitors into the two different published dimer structures. Since some resistance mutations are located in part of the $\mathrm{N}$-terminal region of domain 1 missing in the crystal structures, we produced models of $\mathrm{NS} 5 \mathrm{~A}^{1-191}$ based on the $1 \mathrm{ZH} 1^{10}$ and $3 \mathrm{FQQ}^{11}$ crystal structures together with the $1 \mathrm{R} 7 \mathrm{G}^{19}$ NMR model of the NS5A amphipathic helix (Fig. 1d).

In silico docking of inhibitors to the 3FQQ dimer. In the 3FQQ dimer model, the compounds bind in a well-defined cleft at the dimer interface containing most of the reported BMS-790052 resistance mutation sites, including Y93 and L31. Flexible loops from each monomer, comprising residues 26-32, form part of a hydrophobic pocket into which the prolyl substituents of BMS-790052 bind (Figs. 7, 8e,f). The proposed compound binding site is formed by the following residues: I52, I52*, Q54, Q54*, T56, T56*, P58, P58*, Q62 and Q62* form the "walls" of the site; Y93, Y93*, T95 and T95*are located in the base of the site; K26, L28, P29, R30, L31, P32, V34, S38, I52*, T56, P58, G60, Q62, G96* and P97* form the largely hydrophobic pockets located at either end of the monomermonomer interface $(*$ denotes a residue from the second monomer). 

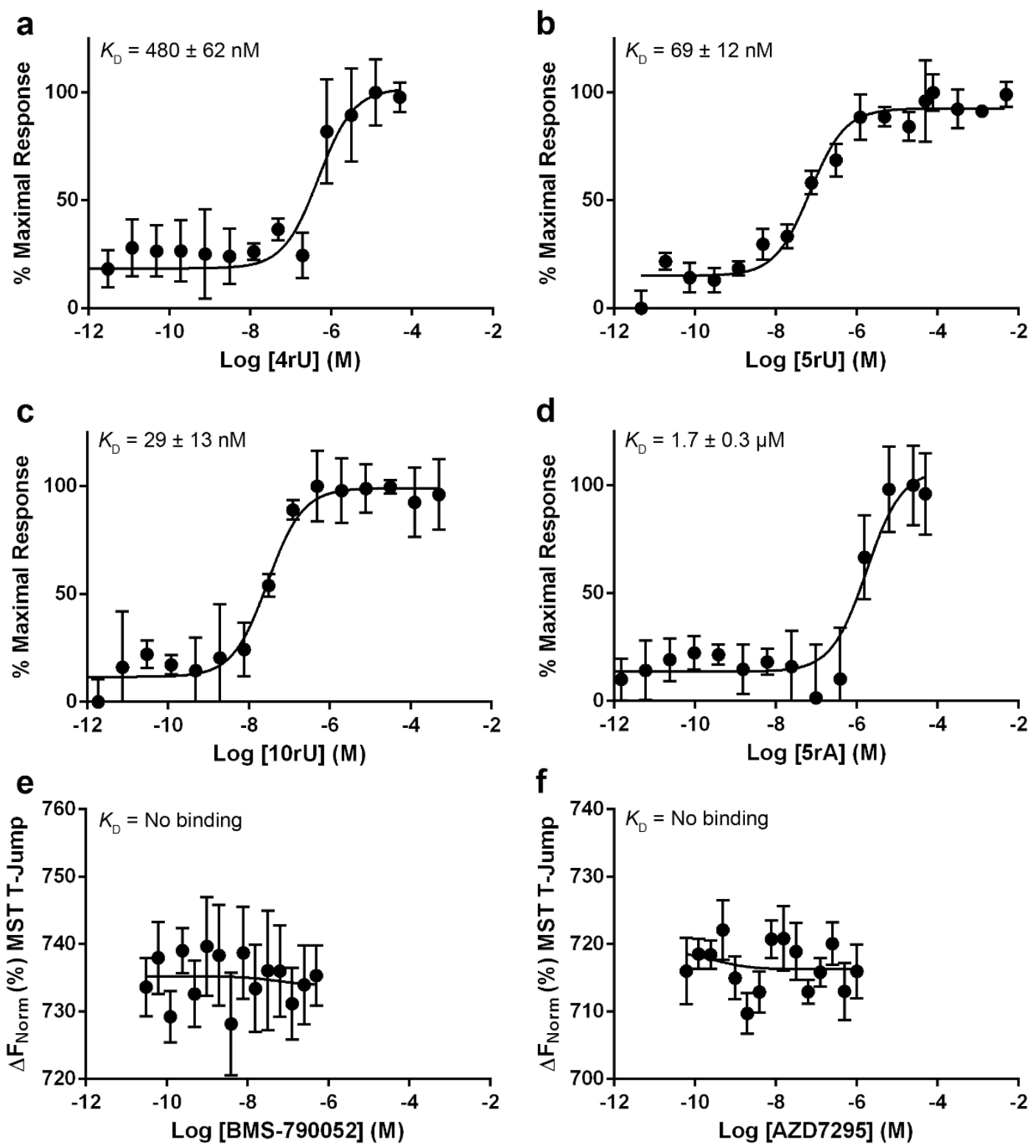

Figure 5 Binding to domain 1 NS5 A6-202 $^{261 V}$ genotype 1 b (con1). Binding of (a) 4rU, (b) 5rU, (c) 10rU, (d) 5rA, (e) BMS-790052 or (f) AZD7295 to NS5A ${ }^{26-202}$ L31V. Values on the Y-axis represent the percentage of the maximal thermophoretic response observed, or in the case of no detectable binding values on the Y-axis represent the thermophoretic shift of the labelled protein. All binding curves were determined in triplicate by MST and are represented as the mean $\pm \mathrm{SD}$.

The docked pose of BMS-790052 shown in Figs. 7 and 8d,e,f is one example of how this compound can bind into the site and the interactions that it can potentially make are: the biphenyl of BMS-790052 sits above and interacts with T95 and T95*; the biphenyl-imidazole rings can interact with I52, I52*, Q54, Q54*, T56, T56*, P58, P58*, Q62 and Q62* (i.e. the "walls"); the imidazole-prolyl rings interact with Y93 and Y93*; each prolyl isopropyl substituent can make hydrophobic interactions with L28, P29 and L31; depending on the prolyl ester group orientation, hydrogen bonds with T56, T56*, Q62 and Q62* are possible.

In silico docking of inhibitors to the $1 \mathrm{ZH1}$ dimer. In the $1 \mathrm{ZH} 1$ dimer model a deep cleft at the interior dimer interface has a positive electrostatic potential and provides a convincing RNA binding site (Fig. 1d). However, in this model, none of the resistance mutations are located in the deep cleft, rather the majority are located on the opposite side of the protein to the interior dimer interface (Fig. 8a).
Hence it seems unlikely that the putative RNA binding site is also the inhibitor binding site.

The inclusion of residues 1-35 into the $1 \mathrm{ZH} 1$ dimer model creates another potential compound binding site, located between the NS5A dimer convex exterior surface and the membrane, which contains most of the known BMS-790052 resistance mutation sites (Fig. 8a,b). The flexible loops, residues 26-32, form the walls of the hydrophobic pocket into which the biphenyl section of BMS-790052 may bind. The possible compound binding site is formed by the following residues: L28, L28*, P29, P29*, R30, R30*, L31, L31*, P32 and P32* form the "walls" of the site; Q54, Q54*, T56, T56*, G60, G60*, Q62, Q62*, Y93, Y93*, T95 and T95* are located on the concave base of the site.

An example of how BMS-790052 can bind into this site is shown in Fig. 8a,b. In contrast to the 3FQQ dimer model, the biphenyl moiety is not in direct contact with NS5A surface residues but interacts with L31 and L31* in the flexible loops. The interaction with NS5A surface residues is confined to the imidazole-prolyl rings and the prolyl 


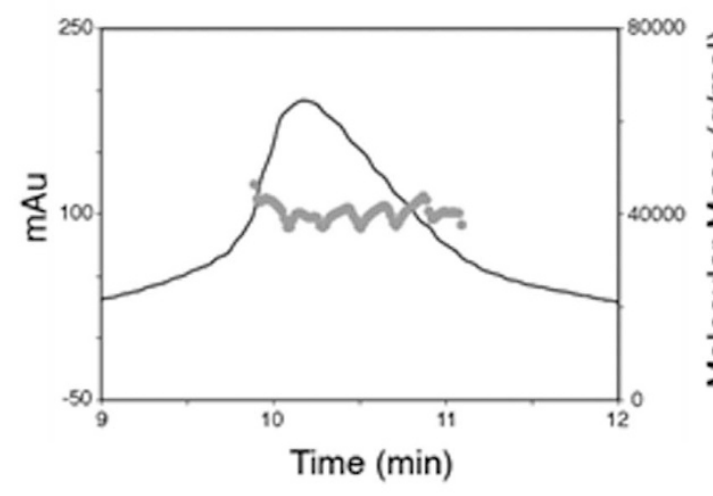

b

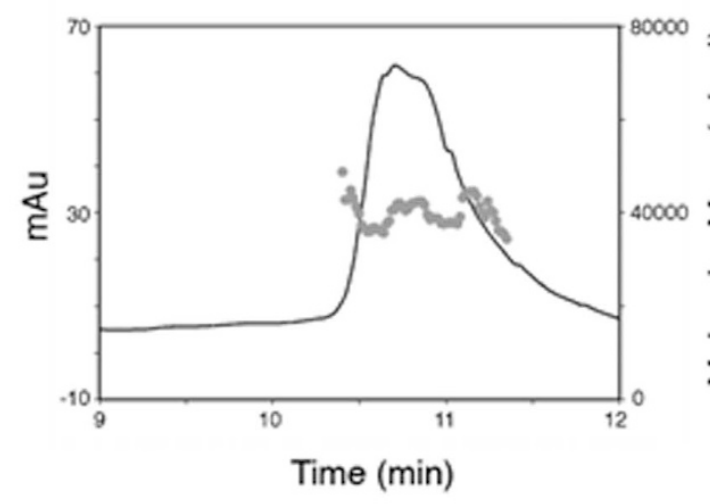

C

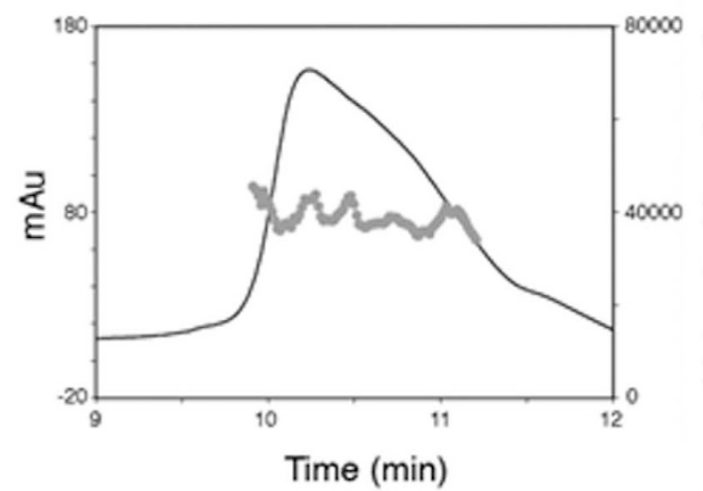

d

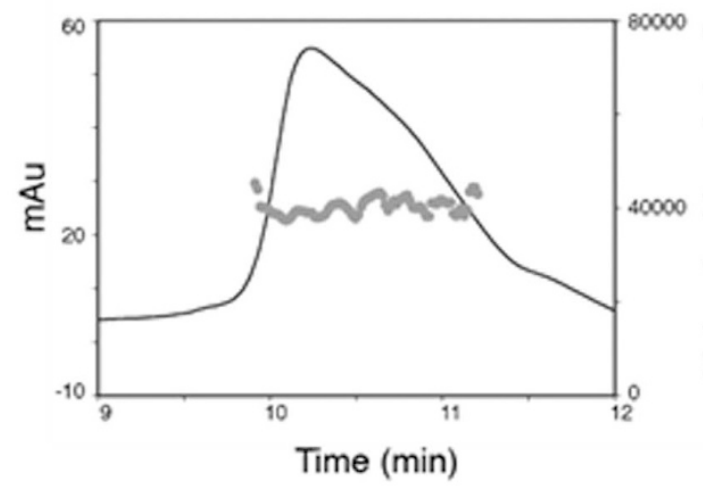

Figure 6 SEC-MALLS analysis of domain 1 NS5A $^{33-202}$ genotype $1 b$ (con1) oligomerisation state. (a) Solution molecular weight analysis of NS5 $\mathrm{A}^{33-202}$ domain 1. (b) Molecular weight analysis in the presence of $5 \mathrm{rU}$, (c) BMS-790052, (d) AZD7295. All molecular weights were determined by MALLS and are shown as grey points, with the black trace representing the absorbance at $280 \mathrm{~nm}$ recorded by an AKTApurifier.

substituents via Q54, Q54*, Q62, Q62*, Y93 and Y93*; as a consequence the compound makes fewer contacts with residues located on the convex surface compared to the $3 \mathrm{FQQ}$ dimer model. Depending on the prolyl ester group orientation, hydrogen bonds with R30, R30*, Q54, Q54*, Q62, Q62*, Y93 and Y93* are possible. Each Y93 lies on the NS5A convex surface at either end of the pocket. Given the flexibility of the loops (residues 26-32) and the concave nature of the protein surface, this site is less convincing than that in the $3 \mathrm{FQQ}$ dimer model.

Like BMS-790052, AZD7295 can also adopt a range of conformations in the proposed compound binding sites of the $3 \mathrm{FQQ}$ and $1 \mathrm{ZH} 1$ dimer models. One example is shown in Fig. 8c. Although both compounds can bind into the same site, the specific interactions made by each inhibitor differ due to their different chemical structures.

Thus the 1ZH1 dimer model provides a suitable RNA binding site without a convincing compound binding site, while the 3FQQ model does the reverse, showing a compound binding site that explains published data without revealing a probable RNA binding site. This leads to the hypothesis that NS5A inhibitors act by favouring a dimeric structure that does not bind RNA.

Mapping genotype differences and resistance mutations onto the NS5 $\mathbf{A}^{1-191}$ dimer models. Any proposed mechanism of action should be consistent with the extensive literature on inhibitor activity against NS5A genotypes and subtypes. Replicon data for the activity of BMS-790052 towards a wide range of NS5A genotypes and subtypes is available. For most genotypes and subtypes, the $\mathrm{EC}_{50}$ is in the range $2-150 \mathrm{pM}$, however notable exceptions are genotype 1a (J1), 2a (J6) and 3a (S52) which have $\mathrm{EC}_{50}$ 's of 1,100, 14,000 and $900 \mathrm{pM}$ respectively $\mathrm{y}^{4,6,28,30-33}$. We mapped the differences between the genotype $1 \mathrm{~b}$ (con1) and genotype 1a (J1), 2a (J6) and 3a (S52) NS5A sequences for residues 26-191 onto the 3FQQ and 1ZH1 dimer models of genotype $1 \mathrm{~b}$ (con1). The residue differences around the proposed compound binding sites for both dimer models (Fig. 8a,f) are as follows: L27 in genotype $1 \mathrm{~b}$ (con1) is replaced by I27 in genotype 3a; L28 is replaced by M28 in genotype 1a and 3a and in genotype 2a it is F28; R30 is replaced by H30 in genotype 1a, K30 in genotype $2 \mathrm{a}$ and A30 in genotype 3a; L31 is replaced by M31 in genotype 2a; I52 is replaced by V52 in genotype 3a; Q54 is replaced by $\mathrm{H} 54$ in genotype 1a, T54 in genotype 2a and S54 in genotype 3a; T56 is replaced by R56 in genotype 1a, 2a and 3a; P58 is replaced by $\mathrm{H} 58$ in genotype 1a; Q62 is replaced by the polar E62 in genotype 1a, N62 in genotype 2a and S62 in genotype 3a; A92 is replaced by C92 in genotype $2 \mathrm{a}$ and in genotype $3 \mathrm{a}$ the analogous residue is E92; T95 and P97 are replaced by E95 and Q97, respectively, in genotype 2a. Many of these genotype $1 \mathrm{a}$ and $1 \mathrm{~b}$ residues (residues 28, 30, 31, 54 and 58) are also mutated in response to replicon treatment with BMS-790052, i.e. they are resistance mutation residues ${ }^{6,34}$.

In genotype $1 \mathrm{~b}$ (con1) the environment around Y93 may play a significant role in preserving the activity of BMS-790052 (depicted in Fig. 8d for the 3FQQ NS5A dimer) compared to genotypes 1a, 2a and 3a. In the 3FQQ NS5A dimer, Y93 is flanked on either side by T56 (from the same NS5A monomer as Y93) and T95* (from the opposing NS5A monomer). As described above, T56 is replaced by the larger charged R56 in genotype 1a (H77), 2a (J6) and 3a (S52) and T95 is replaced by the negatively charged E95 in genotype 2a (J6). Replacing T56 and/or T95* with larger charged residues would prevent BMS-790052 from binding as deeply into the 3FQQ NS5A dimer interface cleft compared to wild type NS5A. In contrast, in the 1ZH1 NS5A dimer model Y93 is flanked by T56 and G33 (all from the same NS5A monomer). Larger charged side-chains at residue 56 are readily accommodated in the 1ZH1 NS5A dimer model 

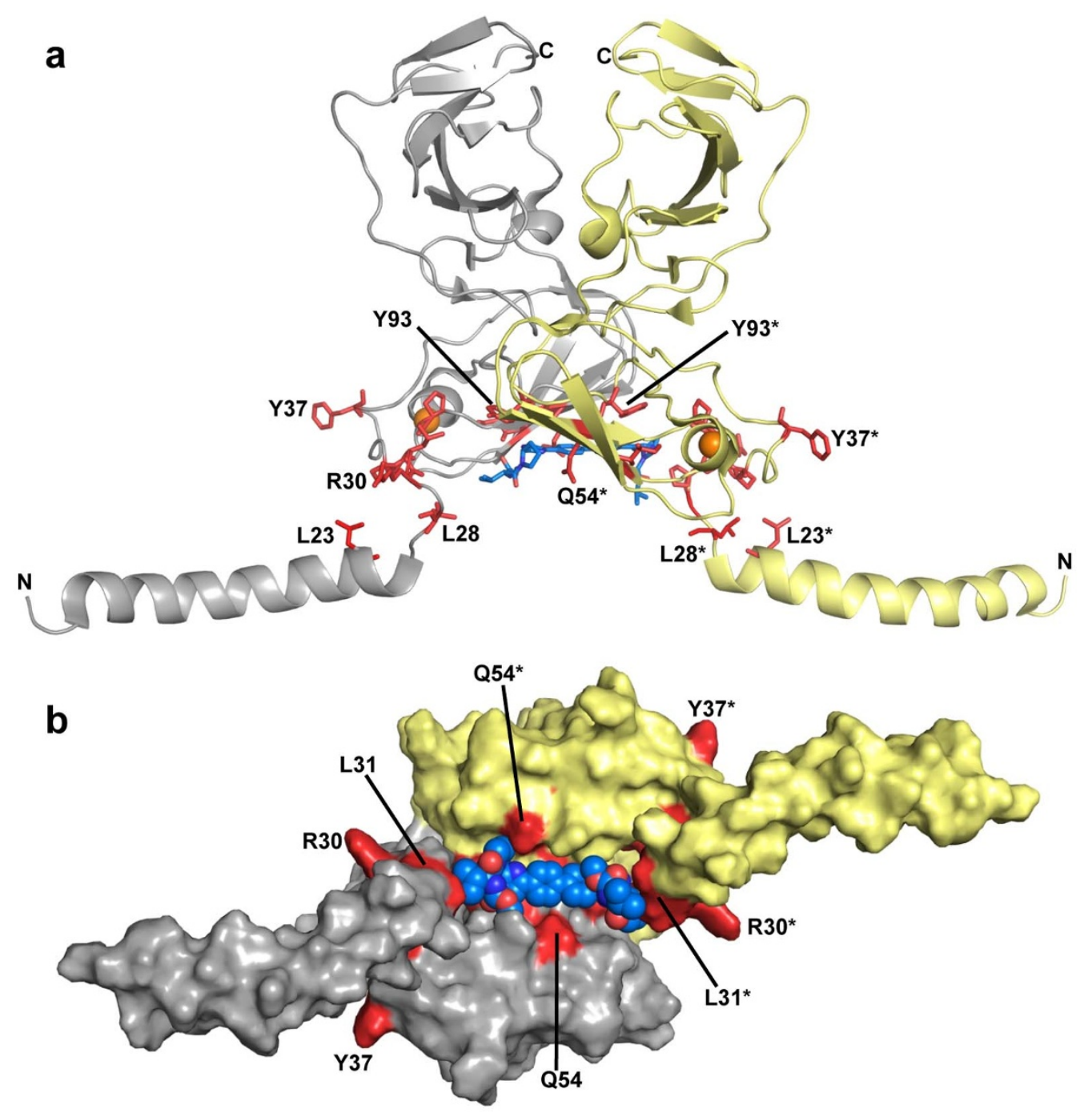

Figure $7 \mid$ Computational docking of BMS-790052 to NS5A ${ }^{1-191}$. (a) The 3FQQ dimer model, coloured as in Fig. 1d. Known BMS-790052 resistance mutation residues for genotype $1 \mathrm{~b}(\mathrm{~L} 23, \mathrm{~L} 31, \mathrm{P} 32, \mathrm{~F} 37, \mathrm{Q} 54, \mathrm{P} 58, \mathrm{I} 63, \mathrm{Y} 93)^{6}$ and genotype 1a (M28, Q30, L31, P32, H54, Y93) ${ }^{6}$ are in red. BMS-790052 (blue sticks) docks into the channel formed by the dimer and interacts with many of the resistance mutation residues. (b) The 3FQQ dimer model shown as a molecular surface. View is a rotation of $-90^{\circ}$ about the X-axis from that shown in (a), looking directly down onto the cleft at the dimer interface. BMS-790052 depicted as blue and red cpk. * denotes residues from the yellow monomer.

without impacting on BMS-790052 binding (G33 is conserved across genotypes).

It has also been observed that the effect of the $\mathrm{Y} 93 \mathrm{H} / \mathrm{C}$ resistance mutation on BMS-790052 replicon activity for genotype $1 \mathrm{~b}$ (con1) is minimal ( $~ 5$-fold decrease in $\mathrm{EC}_{50}{ }^{4}$ ) compared to other genotypes; for example, genotype 1a (H77), 2a (J6) and 3a (S52) exhibit 220fold $^{4}, 143$-fold ${ }^{31}$ and 2,111-fold ${ }^{31}$ decreases respectively. In contrast to the genotype $1 \mathrm{~b}$ (con 1 ) replicon data, a much larger decrease in BMS-790052 binding was observed in the MST in vitro assay ( $>50$ fold decrease in $K_{\mathrm{D}}$ ). In the 3FQQ NS5A dimer, replacing the common resistance site residue $\mathrm{Y} 93$ with histidine (i.e. $\mathrm{Y} 93 \mathrm{H}$ ) enables the side-chain to interact with both T56 (from the same NS5A monomer as $\mathrm{Y} 93 \mathrm{H}$ ) and T95* (from the other NS5A monomer). The $\mathrm{Y} 93 \mathrm{H}$ resistance mutation was modelled in the 3FQQ dimer and, while some of the possible histidine side-chain conformations did not impact on the binding of BMS-790052, other conformations of the histidine side-chain prevented BMS-790052 from binding as deeply into the cleft compared to wild type NS5A (as illustrated in Fig. 8d). In the 1ZH1 NS5A dimer model Y93H is flanked on either side by T56 and G33 (both from the same NS5A monomer as Y93H), and an interaction between $\mathrm{Y} 93 \mathrm{H}$ and $\mathrm{T} 56$ should not impact on the binding of BMS-790052. For genotype $1 \mathrm{~b}$ (con1), the common L31V resistance mutation confers 23-fold resistance against BMS-790052 in replicon assays ${ }^{6}$, whereas in the MST in vitro assay there was no detectable binding of the inhibitor to the L31V NS5A ${ }^{26-20}$ mutant. In both NS5A dimer models, the $\mathrm{L} 31 \mathrm{~V}$ mutation may reduce inhibitor activity by destabilising the conformation of the residue 26-32 loop. In the 3FQQ dimer model the L31V mutation would also enlarge the hydrophobic pocket located at either end of the dimer interface, thereby reducing the potential for the compound to interact with both pockets simultaneously (Fig. 8e).

Overall, the putative inhibitor binding site in the 3FQQ dimer model (Fig. 7) is consistent with the behaviour of the common resistance mutants $\mathrm{L} 31 \mathrm{~V}$ and $\mathrm{Y} 93 \mathrm{H}$ and also with known BMS-790052 activity towards different genotypes. In contrast, the less plausible inhibitor binding site in the $1 \mathrm{ZH} 1$ dimer (Fig. 8a,b) is not well supported by the published resistance and genotype data.

\section{Discussion}

In summary, we have shown that MST can be used to demonstrate and measure the binding of RNA or inhibitors to NS5A in the absence of other proteins, an issue which has complicated the inter- 
a
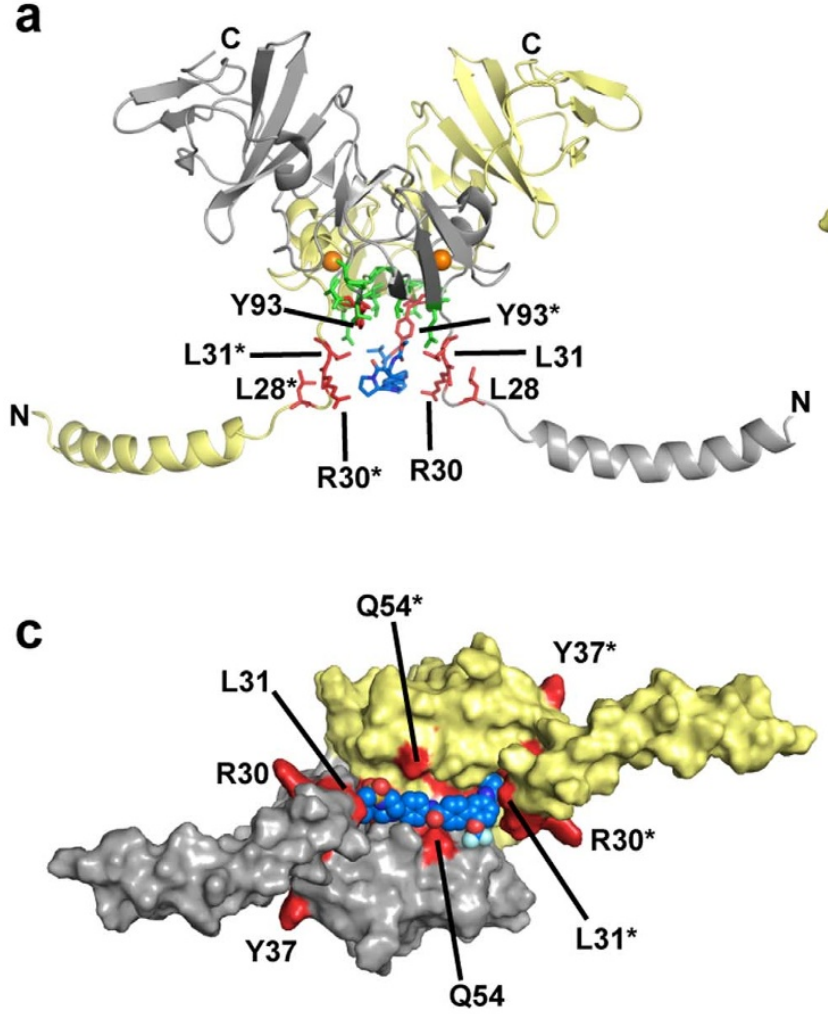

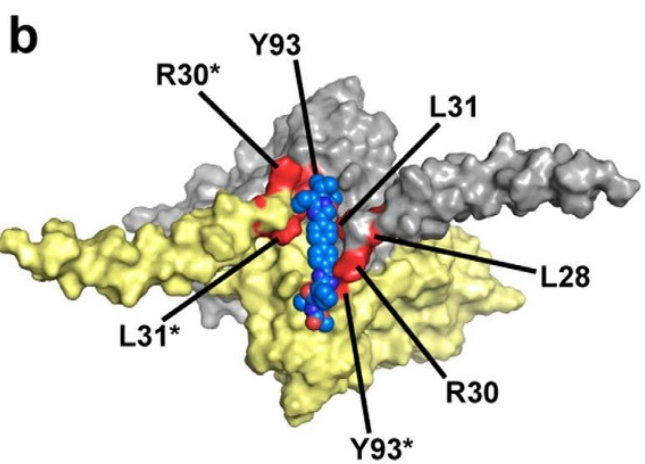

d

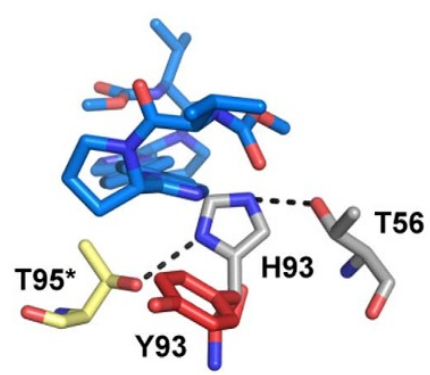

e
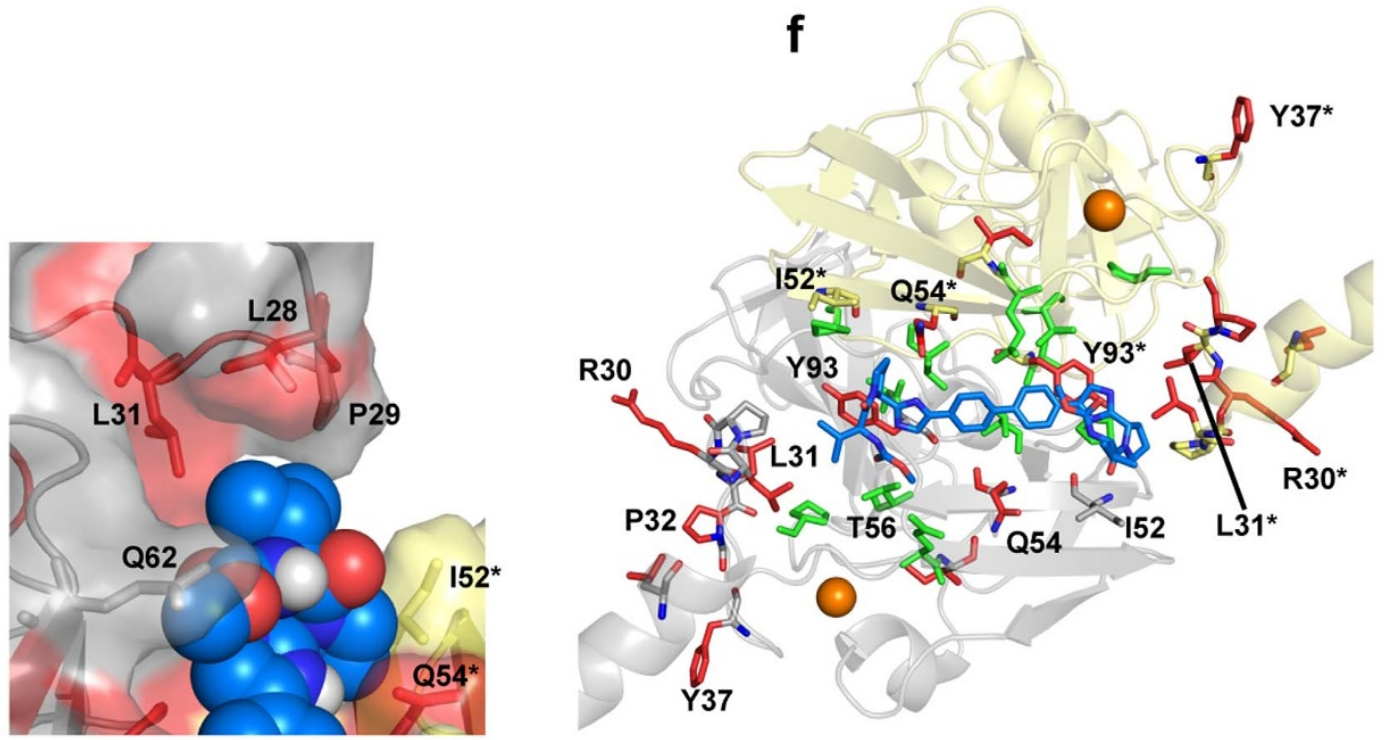

Figure $8 \mid$ Docking of inhibitors and mapping of drug resistance and genotype variation onto the alternate NS5A $\mathrm{A}^{1-191}$ dimer models. The same monomer colour scheme is used as in Figs. 1 and 7. (a) The 1ZH1 dimer model. BMS-790052 (blue sticks) has been docked into a pocket at the dimer interface formed by the concave protein surface (where Y 93 is located) and the two flexible loops consisting of residues $26-32$. The locations of resistance mutation residues (red sticks) and genotype difference residues (green sticks) around the putative inhibitor binding site are indicated. (b) The 1ZH1 dimer model shown as a molecular surface, known resistance mutation residues are coloured red. View is a rotation of $-90^{\circ}$ about the $\mathrm{X}$-axis from that shown in (a) looking directly down onto the pocket at the dimer interface. BMS-790052 depicted as blue and red cpk. (c) AZD7295 (blue, red and cyan cpk) docked into the 3FQQ dimer model (shown as a molecular surface; same view of NS5A ${ }^{1-191}$ as shown in Fig. 7b). Known resistance mutation residues are in red. (d) Model of the 3FQQ dimer Y93H resistance mutation with BMS-790052 docked (blue and red sticks). Showing a close up view of Y93 (red sticks) in wild type and the mutated residue $\mathrm{Y} 93 \mathrm{H}$ (grey and blue sticks). Dashed lines indicate possible hydrogen bonds. (e) Close up view of the 3FQQ dimer model hydrophobic pocket. There are two of these hydrophobic pockets in the 3FQQ dimer model, located at each end of the cleft formed by the monomer-monomer interface. BMS-790052 shown as blue, red and white cpk. NS5 $\mathrm{A}^{1-191}$ shown as cartoon and transparent molecular surface. Sidechains of residues depicted in stick format. (f) Same orientation of BMS-790052 (blue sticks) docked into the 3FQQ dimer model as shown in Fig. 7b; NS5A depicted as a transparent cartoon. The side-chains of resistance mutation residues (red sticks) and genotype difference residues (green sticks) around the putative inhibitor binding site are shown. Other residues interacting with the compound are shown as grey sticks. * denotes residues from the yellow monomer. 
pretation of cell-based approaches. We have shown that both BMS790052 and AZD7295 bind directly to a site located in NS5A domain 1 with nanomolar affinity. Most importantly, we show that RNA binding inhibits compound binding and, conversely, compound binding inhibits RNA binding. Furthermore, we show that common resistance mutants act by directly reducing affinity towards the compounds with little affect on RNA binding. We show that neither RNA nor the compounds affect dimerisation, consistent with previously published observations using different approaches. Finally, computational docking of the inhibitors reveals a compelling compound binding site that is consistent with known resistance mutations and their activity towards different HCV genotypes.

During the preparation of our manuscript O'Boyle and coworkers published $^{7}$ a cell-based study which strongly supports many of our own conclusions. They used chemically labelled (biotinylated and azide cross-linked) stilbene BMS-790052-like compounds in HCV replicon cells to demonstrate tight and specific binding to the NS5A protein. Furthermore, mass spectrometry of labelled NS5A suggested the compounds bound to domain 1 , close to residue L31, which is consistent with our docking results. In addition, cross-linking results suggested the compounds bind close to the dimer interface as we predict from our docking studies.

Our hypothesis for a mechanism of action of NS5A inhibition is that there are distinct RNA binding and inhibitor binding forms of the NS5A domain 1 dimer, represented by the 1ZH1 and 3FQQ dimers, respectively, which are dynamically interconverting. The process is summarised in Fig. 1d. RNA binding traps the NS5A domain 1 dimer in the form that the inhibitor does not bind to, and vice versa. The model provides a molecular basis as to why the common $\mathrm{Y} 93 \mathrm{H}$ resistance mutant does not significantly affect RNA binding (it is located far from the RNA-binding cleft in the 1ZH1 dimer, Fig. 8a,b) but greatly affects inhibitor binding (it is located in the compound binding site in the 3FQQ dimer) (Figs. 7, 8c,d,f). This model also explains why previous attempts to show direct binding using surface plasmon resonance failed ${ }^{12}$, because the technique does not allow free interconversion between dimer states. The advantage of the MST technique is that it is solution-based and allows conversion between NS5A dimer states. Attempts to demonstrate direct binding between NS5A and BMS-790052 using isothermal calorimetry have been unsuccesful ${ }^{12}$, perhaps due to the propensity of NS5A to aggregate readily which can mask titration curves. The computational docking work also explains why compound binding has not been seen by X-ray crystallography: the putative compound binding site in the 3FQQ dimer is buried due to crystal contacts. The model presented here is consistent with the recent FRET studies conducted by Bhattacharya and co-workers ${ }^{12}$ who demonstrated that binding of an NS5A inhibitor in cell culture caused conformational changes in domain 1 resulting in the formation of large NS5A aggregates.

It has been suggested by others that the two types of NS5A dimers may co-assemble into large oligomeric arrays that channel RNA substrates within $\mathrm{HCV}$-infected cells ${ }^{11}$. Furthermore, recent data suggests that BMS-790052 prevents correct NS5A assembly into functional replication complexes ${ }^{35}$. Our evidence for the direct interaction, in solution, of inhibitors with NS5A domain 1 and the potential impact of this interaction on dimer conformation supports this model for the mechanism of action of NS5A protein. BMS-790052 inhibits HCV replicon cells at picomolar concentrations that correspond to one inhibitor molecule for every thousand NS5A molecules. It has been suggested by others that disruption of multimer function by a single inhibitor molecule, via transmission of conformational changes in the same and adjacent polymers, may explain the extraordinary potency of NS5A inhibitors in cells ${ }^{2,4,7}$. The ability of one inhibitor molecule to disrupt the NS5A functional network explains why we see low nanomolar binding for the interaction of BMS-
790052 with NS5A in vitro when others measure picomolar inhibition in cell-based replicon assays.

Many NS5A inhibitors in clinical trials appear similar to the chemical structure of the BMS-790052 compound series ${ }^{2,13}$. Our work in identifying the likely binding site for current inhibitors and the development of a direct binding assay provides a rational framework for refining the molecular mechanism of action, the discovery of entirely novel NS5A inhibitor scaffolds and possible application to related viruses that have NS5A homologues.

\section{Methods}

Materials. BMS-790052 and AZD7295 were gifts from Biota Pharmaceuticals and were synthesised according to published methods ${ }^{36-38}$. In line with the published work on the domain 1 structure $^{10,11}$, we expressed and purified recombinant HCV genotype $1 \mathrm{~b}$ (con 1 ) NS5 $\mathrm{A}^{33-202}$. Briefly, the constructs containing the NS5 $\mathrm{A}^{33-202}$ protein fused to an N-terminal 6-His tag and TEV protease cleavage site were synthesised by Genscript and cloned into pET15b using NdeI and HindIII restriction sites. Protein production was performed in $5 \mathrm{~L}$ baffled flasks containing $2 \mathrm{~L}$ of TB supplemented with $100 \mu \mathrm{g} / \mathrm{mL}$ ampicillin. After inoculation with $20 \mathrm{~mL}$ of an overnight culture, the culture was grown at $37^{\circ} \mathrm{C}$ and $130 \mathrm{rpm}$ in an Inforce shaker for 6 hours, prior to cooling to $16^{\circ} \mathrm{C}$ and induction with $100 \mu \mathrm{M}$ IPTG. The culture was grown overnight at $16^{\circ} \mathrm{C}$ prior to being harvested at $5000 \times \mathrm{g}$ for $20 \mathrm{~min}$. The cell pellets were washed in $\mathrm{PBS}$ and stored at $-80^{\circ} \mathrm{C}$ until ready for purification.

Cell pellets were resuspended in $25 \mathrm{mM}$ Tris, $500 \mathrm{mM} \mathrm{NaCl}, 20 \mathrm{mM}$ imidazole, $10 \%$ glycerol pH 8.0 with Roche Complete EDTA free protease inhibitor cocktail and DNase. Cells were lysed by French Press, and clarified by centrifugation at $20000 \times \mathrm{g}$ for $30 \mathrm{~min}$. The supernatant was then passed over a Ni HiTrap column (GE Healthcare) using AKTApurifier, washed in $25 \mathrm{mM}$ Tris, $500 \mathrm{mM} \mathrm{NaCl}, 20 \mathrm{mM}$ imidazole, $10 \%$ glycerol $\mathrm{pH} 8.0$, and the protein eluted using a gradient up to $25 \mathrm{mM}$ Tris, $500 \mathrm{mM} \mathrm{NaCl}, 400 \mathrm{mM}$ imidazole, $10 \%$ glycerol $\mathrm{pH} 8.0$. The purified protein was dialysed into $25 \mathrm{mM}$ Tris, $500 \mathrm{mM} \mathrm{NaCl}, 20 \mathrm{mM}$ imidazole, $10 \%$ glycerol pH 8.0 and the tag cleaved using an N-terminal 6-His tagged TEV protease. This was then repassed over a Ni HiTrap column to remove the TEV protease and cleaved tag. The protein was then concentrated using a $10 \mathrm{kDa}$ MWCO Amicon concentrator (Millipore) and further purified by size exclusion chromatography using a Superdex 75 26/60 column (GE Healthcare) equilibrated in $25 \mathrm{mM}$ Tris, $250 \mathrm{mM} \mathrm{NaCl}, 10 \%$ glycerol pH 8.0 (Supplementary Fig. S1).

A longer length NS5A construct was explored to characterise the role of other resistance mutation sites outside our original construct: residues 26 to 202 (Supplementary Fig. S1). The Y93H NS5 $\mathrm{A}^{33-202}$ mutant, NS5A $\mathrm{A}^{26-202}$ and the L31V NS5 $\mathrm{A}^{26-202}$ mutant were expressed and purified in a similar manner to NS5 $\mathrm{A}^{33-202}$. Filter-binding assays have previously shown that NS5A binds short stretches of uridine-rich oligonucleotides ${ }^{14,39}$ with the minimum RNA length determined to be five bases. In our study we examined binding to single stranded RNA consisting of four, five or ten uridines (4rU, $5 \mathrm{rU}$ and $10 \mathrm{rU}$ respectively), five adenines (5rA); as well as double stranded RNA formed through the annealing of $5 \mathrm{rU}$ and $5 \mathrm{rA}(\mathrm{ds}(5 \mathrm{rU}))$ (Integrated DNA Technologies).

MST. Solution MST binding studies were performed using standard protocols on a Monolith NT.115 (Nanotemper Technologies). Briefly, recombinant NS5A ${ }^{26-202}$, NS5A $\mathrm{A}^{33-202}$, L31V NS5A ${ }^{26-202}$, Y $93 \mathrm{H}$ NS5 $\mathrm{A}^{33-202}$ or control protein was labelled using the RED-NHS (Amine Reactive) Protein Labelling Kit (Nanotemper Technologies). NS5A was mixed with either RNA, BMS-790052 or AZD7295 with a final buffer condition of $25 \mathrm{mM}$ Tris- $\mathrm{HCl}, \mathrm{pH} 8.0$, containing $250 \mathrm{mM} \mathrm{NaCl}, 10 \%$ glycerol, $0.05 \%$ Tween-20 and 5\% DMSO. Each replicate contained a 16 step 2- to 4 -fold serial dilution series. The protein concentration $(12 \mathrm{nM})$ was chosen such that the observed fluorescence was approximately 1000 units at $40 \%$ LED power. The samples were loaded into hydrophobic capillaries and heated at $40 \%$ laser power for $30 \mathrm{sec}$, followed by $5 \mathrm{sec}$ cooling. The data were normalised against the baseline obtained in the absence of any inhibitor, and the maximal response obtained at the highest concentration of inhibitor. The dissociation constant $K_{\mathrm{D}}$ was obtained by plotting the normalised fluorescence $F_{\text {norm }}$ against the logarithm of the different concentrations of the dilution series resulting in a sigmoidal binding curve that could be directly fitted with a non-linear solution of the law of mass action. All experiments were performed with a minimum of 3 replicates and the normalised fluorescence temperature jump curves were analysed using GraphPad Prism (Version 5, GraphPad, San Diego, CA, USA). $K_{\mathrm{D}}$ 's were compared by one-way Anova with Tukey posttest and $\mathrm{p}<0.01$ was considered to be statistically significant. A Hill slope analysis suggested 1 inhibitor molecule binds per NS5A dimer. No measurable interactions with several unrelated proteins including insulin-regulate membrane aminopeptidase, nicastrin and carbonic anhydrase were observed by MST for either compound. In another control we added excess EDTA to NS5A $\mathrm{A}^{33-202}$ to remove metal from the $\mathrm{Zn}^{2+}$ binding site (leading to destabilisation of the protein fold) and no binding to RNA or either compound was observed.

MALLS. MALLS was carried out both in batch mode using a syringe pump set to $0.1 \mathrm{ml} / \mathrm{min}$ and through size-exclusion chromatography (SEC) using a BioSep-SEC S3000 column (Phenomenex) equilibrated in a buffer containing $25 \mathrm{mM}$ Tris- $\mathrm{HCl}$, $\mathrm{pH}$ 8.0, $250 \mathrm{mM} \mathrm{NaCl}, 10 \%$ glycerol, $0.05 \%$ Tween-20, $5 \%$ DMSO and in the presence 
and absence of $5 \mathrm{mM} \beta$-mercaptoethanol. The column was run at a flow rate of $0.35 \mathrm{ml} / \mathrm{min}$ on an AKTApurifier (GE Healthcare) coupled to a miniDawn MALLS detector and an Optilab T-rEX refractive index detector (Wyatt Technology). The molecular masses of bovine serum albumin (used as a control) and NS5 $\mathrm{A}^{33-202}$ were calculated using a $\mathrm{dn} / \mathrm{dc}$ value of 0.185 . NS5 $\mathrm{A}^{33-202}$ was run at a range of concentrations from $0.3-4 \mathrm{mg} / \mathrm{ml}$. The overall weight-average molar mass for each sample was determined from the concentrations of each molar mass at all points by use of ASTRA 5 software (Wyatt Technologies).

Modelling. The inhibitors BMS-790052 and AZD7295 were constructed and geometrically optimised within SYBYL-X 2.0 (Certara, L. P., http://www.tripos.com) using the MMFF94s forcefield and partial atomic charges, conjugate gradient convergence method. Termination of the optimisation was achieved when the gradient difference of successive steps was $<0.05 \mathrm{kcals} / \mathrm{mol} \bullet \AA$. . Since residues $26-32$, located on a flexible loop connecting the $\mathrm{Zn}^{2+}$ binding domain (residues 33-213) to the amphipathic helix (residues 1-25), is a site of many resistance mutations, we decided to include this region and the amphipathic helix in our models. To construct the 3FQQ dimer model for NS5A ${ }^{1-191}$, residues $1-27$ of the NMR structure $1 R^{2} \mathrm{G}^{19}$ were combined with the 3FQQ A and B chains (residues 33-191) ${ }^{11}$. The loops containing residues $26-32$ were built manually in a conformation that would allow the amphipathic helices (residues 1-25) to interact with the cell membrane and for L28 and L31 to point towards the putative compound binding pocket (Figs. 1d, 7, $8 \mathrm{e}, \mathrm{f})$. The side-chains of the NS5 $\mathrm{A}^{1-191} 3 \mathrm{FQQ}$ dimer model were geometry optimised for 1,000 iterations (or until the gradient of successive iterations was $<0.05 \mathrm{kcal} /$ $\mathrm{mol} \bullet \AA$ ) using the same protocol as above. The $1 \mathrm{ZH} 1$ dimer model (Figs. 1d, 8a,b) was generated in an analogous fashion. The inhibitors were then docked into each of the NS5 $\mathrm{A}^{1-191}$ dimer models using the docking algorithm Surflex (within SYBYL-X 2.0, Certara L. P., http://tripos.com). For both the 3FQQ and 1ZH1 dimer models, the protomol was generated by manually selecting the residues lining the pocket at the monomer-monomer interface, a threshold of 0.50 and a bloat value of 2 . The docking mode used for both NS5 $\mathrm{A}^{1-191}$ dimer models was GeomX and protein flexibility was allowed, all other parameters were at default values. The C-Score scoring function was used and the top 100 ranked poses of each inhibitor were retained for examination. Both BMS-790052 and AZD7295 are conformationally flexible and can adopt a range of orientations within the docking sites of both dimers. The orientations shown in Figs. 7 and 8 are selected examples. Figs. 1, 7 and 8 were produced using PyMOL (http://pymol.org).

1. Ghany, M. G., Nelson, D. R., Strader, D. B., Thomas, D. L. \& Seeff, L. B. An update on treatment of genotype 1 chronic hepatitis $\mathrm{C}$ virus infection: 2011 practice guideline by the American Association for the Study of Liver Diseases. Hepatology 54, 1433-1444 (2011).

2. Belda, O. \& Targett-Adams, P. Small molecule inhibitors of the hepatitis C virusencoded NS5A protein. Virus Res. 170, 1-14 (2012).

3. Suk-Fong Lok, A. HCV NS5A Inhibitors in Development. Clin. Liver Dis. 17, 111-121 (2013).

4. Gao, M. et al. Chemical genetics strategy identifies an HCV NS5A inhibitor with a potent clinical effect. Nature 465, 96-100 (2010).

5. Gane, E. et al. Antiviral activity, pharmacokinetics and tolerability of AZD7295, a novel NS5A inhibitor, in a placebo-controlled multiple ascending dose study in HCV genotype 1 and 3 patients. J. Hepatol. 52, S464 (2010).

6. Fridell, R. A., Qiu, D., Wang, C., Valera, L. \& Gao, M. Resistance analysis of the hepatitis C virus NS5A inhibitor BMS-790052 in an in vitro replicon system. Antimicrob. Agents Chemother. 54, 3641-3650 (2010).

7. O'Boyle, I. D. R. et al. Characterizations of HCV NS5A replication complex inhibitors. Virology doi: 10.1016/j.virol.2013.06.032 (2013).

8. Tripathi, L. P. et al. Understanding the biological context of NS5A-host interactions in HCV infection: a network-based approach. J. Proteome Res. 12 2537-2551 (2013).

9. Targett-Adams, P. et al. Small molecules targeting hepatitis $\mathrm{C}$ virus-encoded NS5A cause subcellular redistribution of their target: insights into compound modes of action. J. Virol. 85, 6353-6368 (2011).

10. Tellinghuisen, T. L., Marcotrigiano, J. \& Rice, C. M. Structure of the zinc-binding domain of an essential component of the hepatitis C virus replicase. Nature 435, 374-379 (2005).

11. Love, R. A., Brodsky, O., Hickey, M. J., Wells, P. A. \& Cronin, C. N. Crystal structure of a novel dimeric form of NS5A domain I protein from hepatitis C virus. J. Virol. 83, 4395-4403 (2009).

12. Bhattacharya, D. et al. Pharmacologic disruption of hepatitis C non-structural $5 \mathrm{~A}$ (NS5A) intra and intermolecular conformations. J. Gen. Virol. doi: 10.1099/ vir.0.054569-0 (2013).

13. Hamatake, R., Maynard, A. \& Kazmierski, W. M. in Annu. Rep. Med. Chem. Vol. 47 (ed Desai Manoj, C.) Ch. 22, 331-345 (Academic Press, 2012).

14. Hwang, J. et al. Hepatitis $C$ virus nonstructural protein 5A: biochemical characterization of a novel structural class of RNA-binding proteins. J. Virol. 84 , 12480-12491 (2010)

15. Lim, P. J. et al. Correlation between NS5A dimerization and HCV replication. J. Biol. Chem. 287, 30861-30873 (2012).

16. Hanoulle, X., Badillo, A., Verdegem, D., Penin, F. \& Lippens, G. The domain 2 of the HCV NS5A protein is intrinsically unstructured. Protein Pept. Lett. 17, 1012-1018 (2010).
17. Liang, Y., Ye, H., Kang, C. B. \& Yoon, H. S. Domain 2 of nonstructural protein 5A (NS5A) of hepatitis C virus is natively unfolded. Biochemistry 46, 11550-11558 (2007).

18. Verdegem, D. et al. Domain 3 of NS5A protein from the hepatitis C virus has intrinsic alpha-helical propensity and is a substrate of cyclophilin A. J. Biol. Chem. 286, 20441-20454 (2011)

19. Penin, F. et al. Structure and function of the membrane anchor domain of hepatitis C virus nonstructural protein 5A. J. Biol. Chem. 279, 40835-40843 (2004)

20. Zillner, K. et al. Microscale thermophoresis as a sensitive method to quantify protein: nucleic acid interactions in solution. Methods Mol. Biol. 815, 241-252 (2012).

21. Lin, C. C. et al. Inhibition of basal FGF receptor signaling by dimeric Grb2. Cell 149, 1514-1524 (2012)

22. Arbel, N., Ben-Hail, D. \& Shoshan-Barmatz, V. Mediation of the antiapoptotic activity of Bcl-xL protein upon interaction with VDAC1 protein. J. Biol. Chem. 287, 23152-23161 (2012).

23. Patnaik, S. et al. Discovery, structure-activity relationship, and biological evaluation of noninhibitory small molecule chaperones of glucocerebrosidase. J. Med. Chem. 55, 5734-5748 (2012).

24. Brvar, M. et al. Structure-based discovery of substituted $4,5^{\prime}$-bithiazoles as novel DNA gyrase inhibitors. J. Med. Chem. 55, 6413-6426 (2012).

25. Bosco, E. E. et al. Rational design of small molecule inhibitors targeting the Rac GTPase-p67(phox) signaling axis in inflammation. Chem. Biol. 19, 228-242 (2012).

26. Lippok, S. et al. Direct detection of antibody concentration and affinity in human serum using microscale thermophoresis. Anal. Chem. 84, 3523-3530 (2012).

27. Carter, M. C. et al. Diphenylcarboxamides as inhibitors of HCV non-structural protein NS5a. Antiviral Res. 86, A19 (2010).

28. Fridell, R. A. et al. Distinct functions of NS5A in hepatitis C virus RNA replication uncovered by studies with the NS5A inhibitor BMS-790052. J. Virol. 85, 7312-7320 (2011)

29. Fridell, R. A. et al. Genotypic and phenotypic analysis of variants resistant to hepatitis $\mathrm{C}$ virus nonstructural protein $5 \mathrm{~A}$ replication complex inhibitor BMS790052 in humans: in vitro and in vivo correlations. Hepatology 54, 1924-1935 (2011).

30. Qiu, D. et al. The effects of NS5A inhibitors on NS5A phosphorylation, polyprotein processing and localization. J. Gen. Virol. 92, 2502-2511 (2011).

31. Scheel, T. K., Gottwein, J. M., Mikkelsen, L. S., Jensen, T. B. \& Bukh, J. Recombinant HCV variants with NS5A from genotypes 1-7 have different sensitivities to an NS5A inhibitor but not interferon-alpha. Gastroenterology 140 , 1032-1042 (2011).

32. Wang, C. et al. In vitro activity of BMS-790052 on hepatitis C virus genotype 4 NS5A. Antimicrob. Agents Chemother. 56, 1588-1590 (2012).

33. Wang, C. et al. In Vitro Activity of Daclatasvir on Hepatitis C Virus Genotype 3 NS5A. Antimicrob. Agents Chemother. 57, 611-613 (2013).

34. Lemm, J. A. et al. Identification of hepatitis C virus NS5A inhibitors. J. Virol. 84, 482-491 (2010)

35. Lee, C. et al. The hepatitis C virus NS5A inhibitor (BMS-790052) alters the subcellular localization of the NS5A non-structural viral protein. Virology 414, 10-18 (2011).

36. Carter, M. C., Cockerill, S., Flack, S. S. \& Wheelhouse, C. J. Heterocyclic derivatives and their use in treating Hepatits C. Great Britain patent WO/2009/ 034390 (2009).

37. Pack, S. K., Geng, P., Smith, M. J. \& Hamm, J. Process for synthesizing compounds useful for treating Hepatitis C. United States patent WO/2009/020825 (2009)

38. Wheelhouse, C. J. et al. Biphenyl derivatives and their using in treating Hepatitis C. Great Britain patent WO/2007/031791 (2007)

39. Foster, T. L., Belyaeva, T., Stonehouse, N. J., Pearson, A. R. \& Harris, M. All three domains of the hepatitis $\mathrm{C}$ virus nonstructural NS5A protein contribute to RNA binding. J. Virol. 84, 9267-9277 (2010).

\section{Acknowledgments}

We thank Biota Pharmaceuticals for gifts of the inhibitors. This work was supported by a capital grant from the Australian Cancer Research Foundation and infrastructure funding from the Victorian Government Operational Infrastructure Support Scheme to St Vincent's Institute. D.B.A. was supported by a Victoria Fellowship from the Victorian Government and the Leslie (Les) J. Fleming Churchill Fellowship from The Winston Churchill Memorial Trust. M.W.P. is a National Health and Medical Research Council of Australia Research Fellow.

\section{Author contributions}

D.B.A. designed and performed the MST binding experiments in Figs. 2-5 and the MALLS experiments in Fig. 6. L.D. also performed some of the MST experiments. T.L.N. did the modelling and computational docking in Figs. 1, 7 and 8. J.W., C.J.M. and M.W.P. designed and supervised various aspects of the study. All authors wrote and revised the paper.

\section{Additional information}

Supplementary information accompanies this paper at http://www.nature.com/ scientificreports 
Competing financial interests: The laboratory where the study took place received funding from anti-infectives company Biota Pharmaceuticals on hepatitis $\mathrm{C}$ projects although not on the studies described in this work. C.J.M. is a Principal Research Associate at St Vincent's Institute and was an employee of Biota Pharmaceuticals. J.W., T.L.N. and M.W.P. were consultants to Biota Pharmaceuticals.

How to cite this article: Ascher, D.B. et al. Potent hepatitis C inhibitors bind directly to NS5A and reduce its affinity for RNA. Sci. Rep. 4, 4765; DOI:10.1038/srep04765 (2014).
This work is licensed under a Creative Commons Attribution-NonCommercialNoDerivs 3.0 Unported License. The images in this article are included in the article's Creative Commons license, unless indicated otherwise in the image credit; if the image is not included under the Creative Commons license, users will need to obtain permission from the license holder in order to reproduce the image. To view a copy of this license, visit http://creativecommons.org/licenses/by-nc-nd/3.0/ 